Hematol 2011;86:928-48.

2. Jawed SI, Myskowski PL, Horwitz S, et al. Primary cutaneous T-cell lymphoma (mycosis fungoides and Sézary syndrome): part I. Diagnosis: clinical and histopathologic features and new molecular and biologic markers. J Am Acad Dermatol 2014;70:205.e1-16.

3. Arafah M, Zaidi SN, Kfoury HK, et al. The histological spectrum of early mycosis fungoides: a study of 58 Saudi Arab patients. Oman Med J 2012;27:134-9.

4. Yamashita T, Abbade LP, Marques ME, et al.

Mycosis fungoides and Sézary syndrome: clinical, histopathological and immunohistochemical review and update. An Bras Dermatol 2012;87:817-28.

5. Jawed SI, Myskowski PL, Horwitz S, et al. Primary cutaneous T-cell lymphoma (mycosis fungoides and Sézary syndrome): part II. Prognosis, management, and future directions. J Am Acad Dermatol 2014;70:223.e117.

\section{Angiosarcoma of the Breast Masquerading as Hemangioma: Exploring Clinical and Pathological Diagnostic Challenges}

Jordan D Frey ${ }^{1}$, Pascale G Levine ${ }^{2}$, Farbod Darvishian ${ }^{2}$, Richard L Shapiro ${ }^{3}$

${ }^{1}$ Institute of Reconstructive Plastic Surgery, ${ }^{2}$ Department of Pathology, ${ }^{3}$ Department of Surgery, New York University Medical Center, New York, NY, USA

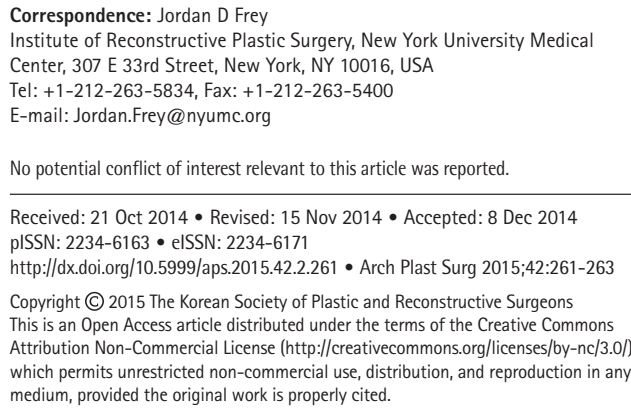

No potential conflict of interest relevant to this article was reported.

Received: 21 Oct $2014 \bullet$ Revised: 15 Nov 2014 • Accepted: 8 Dec 2014 pISSN: 2234-6163・ elSSN: 2234-6171

http://dx.doi.org/10.5999/aps.2015.42.2.261 • Arch Plast Surg 2015:42:261-263

Copyright (C) 2015 The Korean Society of Plastic and Reconstructive Surgeons This is an Open Access article distributed under the terms of the Creative Commons Attribution Non-Commercial License (http://creativecommons.org/licenses/by-nc/3.0/) which permits unrestricted non-commercial use, distribution, and reproduction in any medium, provided the original work is properly cited.

Vascular tumors of the breast are very rare.

Angiosarcomas account for only $0.04 \%$ of all breast tumors and $3 \%$ to $9 \%$ of breast sarcomas and may be primary or, more commonly, secondary [1-5]. Primary angiosarcomas of the breast require a high degree of clinical suspicion for accurate diagnosis and appropriate treatment. Even so, they may be misdiagnosed and treatment must be thoughtfully directed with this possibility in mind. We present a case report illustrating the challenges to accurate and timely diagnosis of primary angiosarcoma of the breast despite thorough preoperative work-up.

The patient is a 44-year-old nulliparous woman with no significant past medical or surgical history. Her most recent mammogram demonstrated only bilateral fibrocystic disease. She presented to one of the authors with a two month history of a rapidly enlarging right breast; most notably with deformation and fullness of the superior pole. An ultrasound performed in the office revealed a $15-\mathrm{cm}$ lesion of the right breast and subsequent fine-needle aspiration (FNA) was performed. The aspiration biopsy showed dissociated and clustered bland spindle cells. Some tumor cells exhibited mild cytologic atypia with intracytoplasmic metachromatic material which may represent degenerated red blood cells, and nuclear membrane irregularities (Figs. 1, 2). The diagnosis was that of a spindle cell lesion.

A subsequent core biopsy showed areas of vascular proliferation with individual channels isolated from one another; there were also areas of anastomosing vascular spaces lined by mildly pleomorphic endothelial cells with rare mitotic figures (Fig. 3). A diagnosis of hemangioma was made.

A magnetic resonance imaging (MRI) confirmed a $6.6 \times 6.3 \times 7.4 \mathrm{~cm}$ enhancing heterogeneous, hyperintense mass on T1- and T2-weighted images in the right breast with superficial feeding vessels and skin contact without intramuscular extension (Fig.

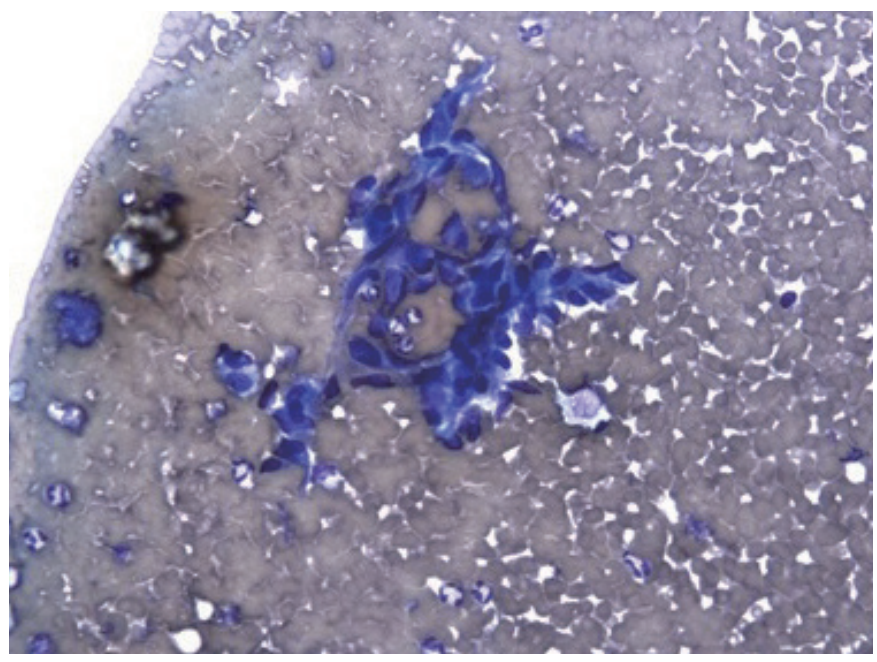

Fig. 1.

Aspiration biopsy (Diff Quik stain, $\times 20$ ). Cluster of mildly pleomorphic spindle cells in a background of blood. 


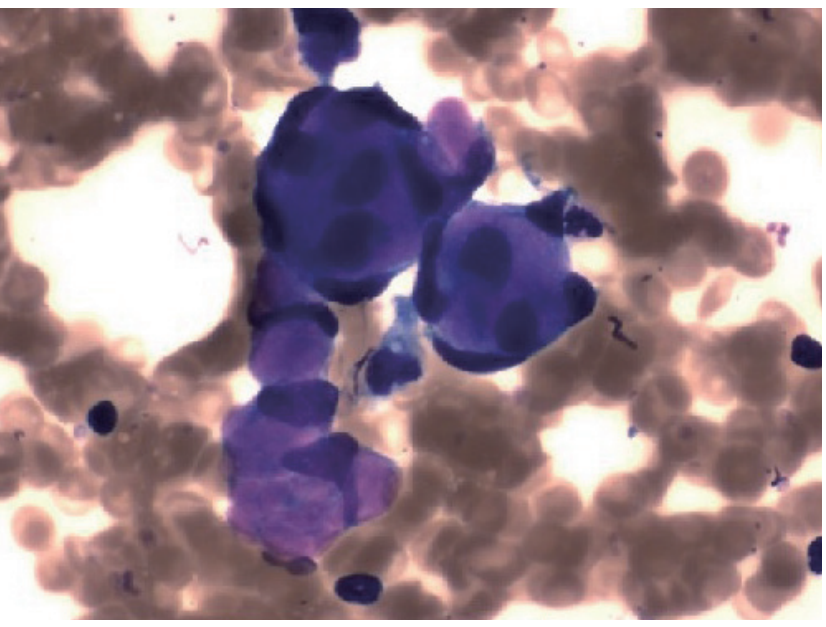

Fig. 2.

Aspiration biopsy (Diff Quik stain, $\times 40$ ). Microacinar groups of spindle and epithelioid cells associated with scant intracytoplasmic metachromatic material.

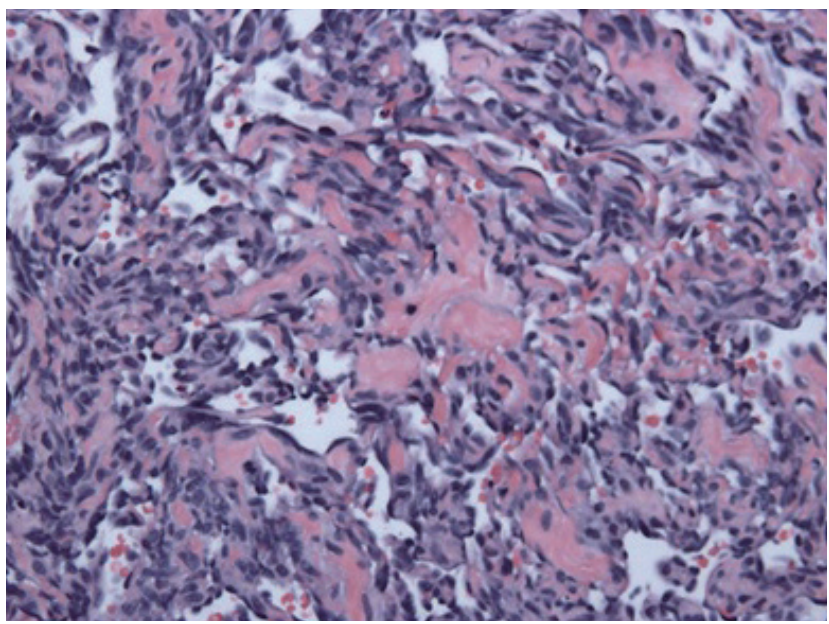

Fig. 3.

Core biopsy $(H \& E, \times 20)$. Anastomosing vascular channels lined by bland spindle cells.
4). The following day, she underwent right partial mastectomy with excision of the mass with a margin of surrounding breast tissue. A partial mastectomy was performed in place of simple excision of the presumed hemangioma due to the size of the lesion and the possibility of malignancy despite imaging and biopsies suggesting otherwise. Intraoperatively, the mass was noted to be heterogeneously solid and cystic. A lymph node in the tail of Spence was biopsied. A large defect was created by the resection requiring mobilization of a medial pillar of breast tissue to fill the defect with good cosmetic outcome.

Final pathology revealed an ill-defined infiltrative $9.0 \mathrm{~cm}$ tumor composed of inter-anastomosing channels and blood lakes lined by atypical cells including focal hobnail cells. There were multiple solid areas of tumor demonstrating a high mitotic rate (up to $25 / \mathrm{mm}^{2}$ ). No tumor necrosis was identified. Immunostains for CD31, CD34, and ERG

highlighted the tumor cells in the solid areas as well as the cells lining the interanastomosing channels confirming the endothelial differentiation of the tumor. Overall, the findings were diagnostic of angiosarcoma, grade 3. Anterior, superior and inferior margins were positive for malignancy. The lymph node was negative for sarcoma.

This case is unique for a variety of reasons. While the size and growth of the mass presented was more consistent with angiosarcoma than hemangioma, clinical evaluation is non-specific. Often, patients have only a painless mass with a bluish hue only as in this case [1], leaving physicians to rely on radiological and pathological data for diagnosis prior to definitive excision.

This patient's initial diagnosis of benign hemangioma was predicated on multiple imaging and tissue studies. When found on mammography, angiosarcomas present as poorly defined masses without calcifications with $19 \%$ to $33 \%$ being mammographically occult [1], as in the patient's case. Ultrasound findings in breast hemangiomas demonstrate heterogeneous echogenecity without acoustic shadowing [1]. While the patient's ultrasound imaging demonstrated mixed echogenicity with both peripheral and central vascularity, acoustic shadowing was present and the vascularity was not uniform throughout the lesion. An MRI was obtained to better delineate the lesion's extension within the breast. MRI typically demonstrates a hypointense lesion on T1-weighted images and a hyper-intense lesion on T2-weighted images [5]. The patient's MRI results, however, demonstarted hyperintense lesions on $\mathrm{T} 1$ - and $\mathrm{T} 2$-weighted images without extension into the pectoralis fascia.

FNA results of vascular tumors of the breast are largely indeterminate and may show hypocellularity; however, it is often not adequate to differentiate malignant from benign vascular tumors. The patient's FNA showed an indeterminate spindle cell lesion. $\mathrm{CNB}$ is favored in the diagnosis of breast vascular tumors as it is touted to definitively differentiate benign and malignant lesions. Despite an adequate sample, CNB incorrectly identified the lesion as capillary hemangioma. Cellular markers of angiosarcoma are not useful since they simply demonstrate the endothelial (vascular) nature of the 
lesion; a feature both lesions share (personal communication). As illustrated in this case, the diagnosis of angiosarcoma requires a high degree of clinical suspicion and is very difficult to differentiate from benign hemangiomas [1], a capillary hemangioma in this case. Both confirmatory imaging and tissue studies contributed to the misdiagnosis on initial presentation.

The treatment of benign hemangiomas of the breast is simple excision whereas treatment of angiosarcoma requires complete local excision with margins or total mastectomy [1]. Mixed results have suggested that radical surgical intervention does not offer a significant survival advantage compared to conservative treatment. Despite the tissue diagnosis of a benign lesion, the mass was removed with a cuff of normal breast parenchyma with intent to remove all visible tumor with margins given its large size. While the role of adjuvant chemoradiation in the treatment of angiosarcoma of the breast remains controversial, she has elected for conservative expectant management and will forego adjuvant therapy at this time.

We present the case of a 44-year-old woman with a rapidly enlarging unilateral breast mass initially diagnosed through imaging and tissue biopsy as a capillary hemangioma, but found to be grade III primary angiosarcoma on surgical excision. This case illustrates the importance of clinical suspicion in vascular tumors of the breast as imaging and tissue studies may be indeterminate and misleading. When clinical suspicion remains high despite biopsy results, complete excision with margins or mastectomy should be undertaken while awaiting final pathology.

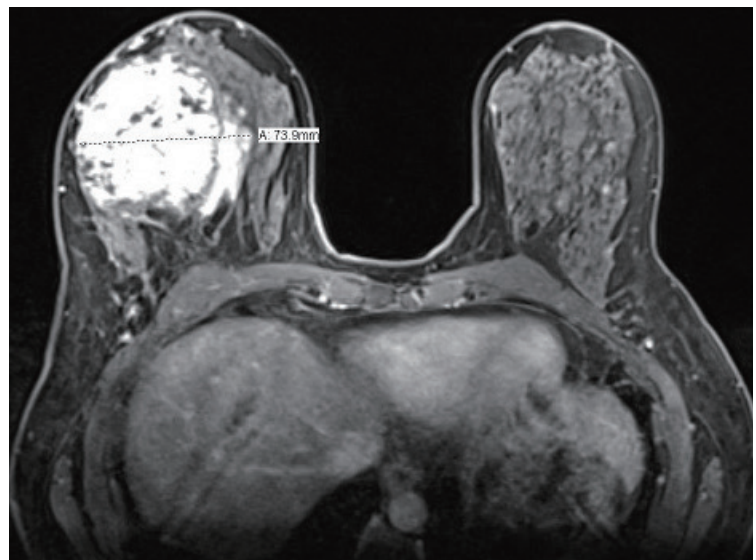

Fig. 4.

Axial post-contrast magnetic resonance imaging of right breast lesion.

\section{References}

1. Brodie C, Provenzano E. Vascular proliferations of the breast. Histopathology 2008;52:30-44.

2. Kaklamanos IG, Birbas K, Syrigos KN, et al. Breast angiosarcoma that is not related to radiation exposure: a comprehensive review of the literature. Surg Today 2011;41:163-8.

3. Scow JS, Reynolds CA, Degnim AC, et al. Primary and secondary angiosarcoma of the breast: the Mayo Clinic experience. J Surg Oncol 2010;101:401-7.

4. Conde DM, de Paula EC, Jales RM, et al. A clinically palpable cavernous hemangioma of the breast in an 80year old woman. Eur J Obstet Gynecol Reprod Biol 2013;167:236-7.

5. O'Neill AC, D'Arcy C, McDermott E, et al. Magnetic resonance imaging appearances in primary and secondary angiosarcoma of the breast. J Med Imaging Radiat Oncol 2014;58:208-12. 